\title{
Role of Distribution Function in Vibration Related Error of Strapdown INS in Random Vibration Test
}

\author{
A. Abdoli* \\ Faculty of Electrical Engineering, Iran University of Science and Technology, Tehran, Iran
}

\section{S. H. Taghavi**}

Faculty of Computer and Electrical Engineering, Shahid Rajaee University, Tehran, Iran

\begin{abstract}
In this paper, a detailed investigation of the random vibration test is presented for strapdown inertial navigation systems (INS). The effect of the random vibration test has been studied from the point of view of navigation performance. The role of distribution functions and RMS value is represented to determine a feasible method to reject or reduce vibration related error in position and velocity estimation in inertial navigation. According to a survey conducted by the authors, this is the first time that the effect of the distribution function in vibration related error has been investigated in random vibration testing of INS. Recorded data of navigation grade INS is used in offline static navigation to examine the effect of different characteristics of random vibration tests on navigation error.
\end{abstract}

Key words: Strapdown INS, random vibration test, distribution function, RMS value

\section{Introduction}

An inertial navigation system is a set of instruments that can be used to determine the position and velocity of a vehicle solely through inertial measurements. Currently prevalent inertial instruments are accelerometers and gyroscopes. The position and velocity of a moving object can be characterized by the specific arrangements of inertial sensors assembly (ISA), as well as by analysis of inertial navigation equations in the navigation computer. ISA is a structure containing multiple inertial sensors in a fixed orientation relative to one another [1].

Stable platform and strapdown are two types of navigation systems. In a stable platform system, the inertial sensors are isolated from the rotations of the moving object so that they can be maintained at a specific orientation relative to the earth or an inertial space. In this system, computations of velocity and position are relatively simple and better performance is expected from gyroscopes as they should measure very small angular rates. Gyroscopes in a strapdown INS measure the full rotation rates of a moving object and keep track of the instantaneous orientation of accelerometers in order to accurately integrate the accelerations into evaluations of velocity and position. More high-tech gyroscopes are therefore necessary in a strapdown INS. Strapdown navigation requires the use of complex computations, and this technology has been made possible by the development of faster and smaller digital processors.

During all-inertial navigation, the inertial sensors are the only instruments used for calculating linear and angular motion. As there is no correction by external sources, navigation error is bound to increase exponentially over time. Therefore in all-inertial navigation, accurate position and velocity measurements can only be determined using precise INS. Although accurate sensors and precise calibration are essential, reliance on these factors is not sufficient.

INS is generally required to operate in an extensive variety of environments. Therefore, it is imperative to evaluate how
This is an Open Access article distributed under the terms of the Creative Commons Attribution Non-Commercial License (http://creativecommons.org/licenses/by$\mathrm{nc} / 3.0 /$ which permits unrestricted non-commercial use, distribution, and reproduction in any medium, provided the original work is properly cited. (cc) * Graduate Student

** Graduate Student, Corresponding author : hossein_taghavi@hotmail.com 
well the INS works under conditions that simulate the environment in which the INS needs to navigate. There are a series of routine tests that INS should pass in order to evaluate mechanical strength or electrical rigidity of equipment and sensors. It is also important to investigate how these tests that simulate in-flight situations may affect navigation performance. In other words, an INS needs to be tested for robustness in relative environmental conditions to determine how they influence the navigation performance. High and low temperature, vacuum, shock and vibration are the main environmental conditions that require testing. Among these tests, the random vibration test has a significant destructive effect on navigation performance, compared to other tests.

Calibration of INS is conducted by means of an accurate two or three axis turn-table. The table places the INS in different positions and rotates it at various angular rates to stimulate the accelerometers and gyroscopes of INS by forces such as the earth's rotation, gravity and finally, the rotation of the turn-table. By using a chamber or variable temperature stabilization, thermal calibration is possible. As vibratory calibration is impossible, assessing the robustness of inertial sensors and other issues such as bias drift and vibration rectification errors are essential to determine the vibration related error [2]. First of all, it is necessary to explain why a random vibration test is required, and how it should be conducted.

During environmental testing of INS, it is easier to determine its sensitivity by executing static navigation. For random vibration tests, INS is mounted on the electromagnetic shaker and outputs are stored in a computer via serial port. The recorded data will be used in offline static navigation to investigate the effect of random vibration [3].

According to accessible telemetry data of flying objects, it is obvious that there is a high frequency, low amplitude variation in sensor output. Among all the possible sources that may be responsible for this effect, random vibration is the most likely. Environmental situations as well as the mechanical characteristics of flying objects such as turbulence and mass variation, motor or turbine operation, sudden decrement in mass, changes in fuel flow or air pressure variation can vibrate the structure and INS . Therefore, it is necessary to inspect the robustness of a calibrated INS relative to vibration in cases of mechanical behavior and increments in navigation error; the latter is addressed in the scope of this study. In other words, a random vibration test is required to simulate the vibration behavior of the environment in order to evaluate vibration related error and quality of sensor isolation, with the restrictions of laboratory testing taken into consideration.
In the following section, the random vibration test and its principles are discussed. Afterward, there is an investigation of output signals in the presence of random vibration with different approaches, and analytical reasoning is presented for the proposed claims. In the last section, an experimental test and analysis is performed for two navigation grade strapdown INS blocks to confirm the methodical analysis.

\section{Random Vibration Test}

As mentioned before, an assembled and sealed INS block should pass particular environmental tests. This paper is focused on the random vibration test, which is a severe test and may cause lasting damage to sensors or electronics equipment so it is usually the last set of tests performed [4]. The purpose of this test is to investigate the resonant and frequency responses of mounted sensors.

\subsection{Test Equipment}

Vibrating INS with particular characterizations requires a specific device. A controllable shaker enables the application of various forms of vibratory motions to an INS. A linear shaker is used in these tests for its ability to apply vibration to the INS on each side of a single axis. It is important that the table and fixture be level, because any misalignment in the axis of the table, fixture, or sensor mounting will cause a nonzero output, even in a static condition. This minor output is increased by the effect of vibration, and could be a major source of error.

Even the most precise linear shakers have their own problems. Such problems are usually caused by structural resonances and large cross-axis vibration levels in addition to angular vibration inputs and offsets [5]. In this study, the vibration testing system applied is the $i$-series from IMV, which can apply vertical vibration in the " $\mathrm{X}$ " axis, and has extra slip configuration for applying vibration in the " $\mathrm{Y}$ " and "Z" axes of INS. Apart from a precise shaker, the fixture is also important. The structure of the fixture may vary according to requirements, but all of them share some common characteristics. Fixture resonance and cross coupling effects are the most important considerations that can affect vibration behavior, and they do so by boosting it. An optimal fixture is the one with the lowest natural frequency at about $50 \%$ higher than the highest required forcing frequency [6]. Also, the structure, stiffness and material of the fixture should all be well engineered. The applied fixture should have a satisfactory quality of manufacturing and an accurate design, which perfectly matches the requirements. 


\subsection{Test Specifications}

Random vibration is a test required for industrial and military manufacturers of electronic equipment, because random vibration is more closely related to the actual environment in which the electronic equipment must operate. When electronic equipment is subjected to a random vibration test over a frequency bandwidth of $2 \mathrm{~Hz}$ to 2 $\mathrm{KHz}$, all of the structural resonances of the electronic system within the same bandwidth will be excited at once. This is different from sinusoidal vibration, in which excitation is executed individually over a bandwidth. Random vibration curves are mostly expressed in white noise curves [6], as shown in Fig. 1. This figure shows the actual input curve that the INS will experience in this study. The curve is expressed in $G^{2} / H z$ along the vertical axis and the frequency is plotted along the horizontal axis. $\mathrm{G}^{2} / \mathrm{Hz}$ is the unit of power spectral density, $\mathrm{P}$, and is defined as,

$$
P=\lim _{\Delta f \rightarrow 0} \frac{G^{2}}{\Delta f}
$$

where $G$ is RMS acceleration, expressed in the unit of gravity and $\Delta f$ is the bandwidth of the frequency range. RMS acceleration can be obtained by integrating the area under the output random vibration curve, then computing the square root of the area, which is determined as:

$$
\sqrt{\text { Area }}=\sqrt{\frac{G^{2}}{H z} \times H z}=\sqrt{G^{2}}=G_{R M S}
$$

Eq. (2) is independent of the curve shape; however, as random vibration input curves are plotted in log-log coordinates, slopped sections of the curve should be calculated according to Eq. (3) [6].

$$
A=\frac{3 P_{2}}{3+S}\left[f_{2}-\left(\frac{f_{1}}{f_{2}}\right)^{\frac{s}{3}} f_{1}\right]
$$

In Eq. (3), the parameters $P_{2}, f_{1}$ and $f_{2}$ are similar to what

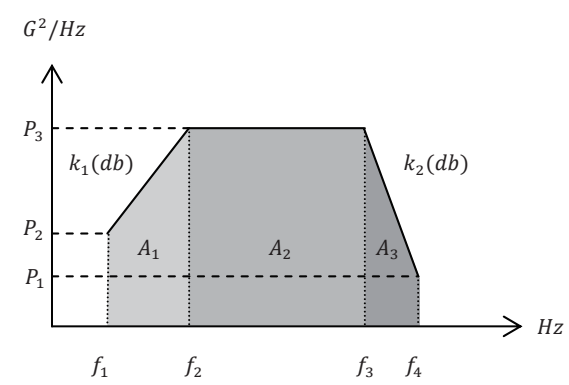

Fig. 1. Random vibration input PSD curve (profile) is shown in Fig. 1 , and $s$ is gradient in $\mathrm{dB}$ unit $\left(k_{i}\right.$ in Fig 1$)$. So, for computing the RMS acceleration experienced by INS during random vibration test with a curve similar to the one proposed in Fig. 1, the following formula will be used,

$$
G_{R M S_{\text {total }}}=\sqrt{A_{1}+A_{2}+A_{3}}
$$

where $A_{1}$ and $A_{3}$ are calculated using Eq. (3) and $A_{2}$ is achieved by a simple area calculation. Although the magnitude of the acceleration level is important, the magnitude of input PSD levels in the region of the structure's resonant frequencies is even more important.

For the applied test, $P_{1}, P_{2}, P_{3}, k_{1}, k_{2}$ and $f_{1}$ to $f_{4}$ are known. Total $G_{R M S}$ is therefore calculable and is equal to $6.105 \mathrm{~g}$. This RMS acceleration is what excites the INS; but accelerometers sustain less acceleration because of mechanical dampers that isolate them from the INS body. These mechanical dampers reduce the RMS acceleration sensed by the sensors. For inertial navigation, it is necessary to equip accelerometers with a mechanical filter to eliminate high frequencies [7].

For example, a random vibration test with a profile such as the one shown in Fig. 1 was performed in the " $\mathrm{X}$ " axis of the INS. Using calibration coefficients, data recorded by the accelerometer showed an RMS value of $4.372 \mathrm{~g}$ in the positive channel, and $3.376 \mathrm{~g}$ in the negative channel, while the nominal value of applied acceleration was $6.102 \mathrm{~g}$ RMS. This decrement was caused by mechanical dampers. It should be noted that approximately $1 \mathrm{~g}$ RMS difference between positive and negative channels should be attributed to the effect of the earth's gravity, and was applied to the positive channel at the time of testing.

In order to predict the level of probable acceleration in a random vibration test, it is necessary to be aware of probability distribution function (PDF). The applied shaker has a customizable Gaussian distribution. It is defined by,

$$
Y=\frac{e^{-\frac{X^{2}}{2 \sigma^{2}}}}{\sigma \sqrt{2 \pi}}
$$

The right side of Eq. (5) represents probability per unit of $X$, for the ratio of instantaneous acceleration $(X)$ to the RMS acceleration $(\sigma)$. The instantaneous acceleration will pass the $1 \sigma$ value, which is equal to RMS value, in $31.7 \%$ of the test period. It will pass the $2 \sigma$ value, which is twice the RMS value, in $4.6 \%$ of the test period. It will pass the $3 \sigma$ value, which is three times the amount of RMS value, in only $0.27 \%$ of the test period. Although higher acceleration levels of $4 \sigma$ and $5 \sigma$, which are respectively equal to 24.4 and $30.5 \mathrm{~g}$, can sometimes be expected in a defined test, they are usually ignored because the test equipment has $3 \sigma$ clippers built into its control systems. More information about random 
vibration test is presented in [8-9].

Therefore, the two main specifications for a random vibration test are RMS value and distribution function. Hereafter, the effect of both factors will be examined.

In industrial laboratories, random vibration test profiles typically have higher acceleration levels than values in the real world environment. For example, an input level of $6.0 \mathrm{~g}$ RMS is applied for one minute to replicate the same amount of damage that can be expected from $1.5 \mathrm{~g}$ RMS over a week in the real world environment. These commitments belong to the qualification tests that challenge the physical strength of sensors and electrical equipment. Investigating the vibration related error in navigation requires a simpler profile. Several test profiles are addressed in [10-12].

\section{Effect of Random Vibration}

Random vibration, by excitation of nonlinear terms and drifting in the sensor's bias, invalidates calibration coefficients and imposes a great error in navigation that increases over time. In [13-14] compensation of drift for fibre optic gyroscopes (FOG) was examined. In [15] the wavelets technique was applied to detect and extract vibration disturbance from inertial measurement units. In this study, the random vibration test will be examined in terms of navigation error (vibration related error).

Figures 2 and 3 show the effects of random vibration on the accelerometer and gyroscope output pulses in the " $\mathrm{X}$ " axis, while vibration is applied in the INS " $\mathrm{X}$ " axle. Comparison between the vibration signal and the vibration-free static signal, as well as a frequency spectrum analysis, confirms that random vibration entirely changes the characteristics of output signals. This presents the question of which properties of the random vibration test influence navigation error?
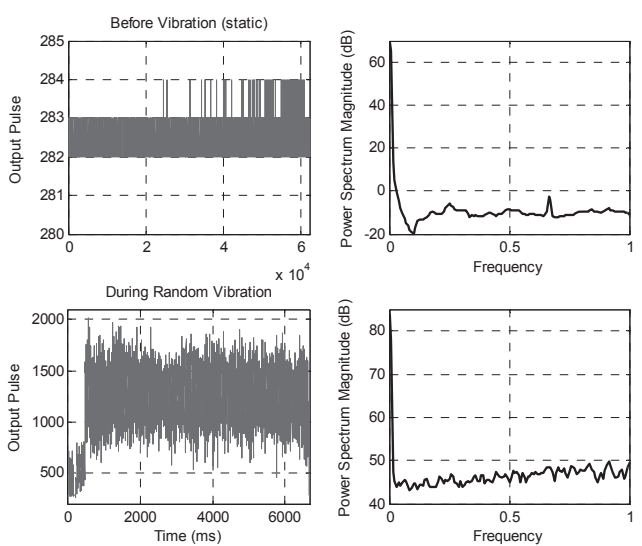

Fig. 2. Effect of random vibration test with profile similar to Fig. 1 on positive channel of accelerometer $\mathrm{X}$
RMS value and distribution function are two main characteristics that define a random vibration test. To investigate the effect of the distribution function, two tests must be applied with equal RMS values and different PDFs. This is possible by customizing the shaker's controller program. The standard deviation of the shaker's Gaussian distribution function, $\sigma$ in Eq. (5), was reduced in order to produce the second PDF. This change affects both the magnitude and scattering of instantaneous outputs. Changing the distribution function in the shaker's controller should not change the mean value of output pulses. Since there is no access to the shaker output signal, the output of accelerometer $\mathrm{X}$ was recorded in order to investigate the effect of $\sigma$ variation (Fig. 4). The PDF of different outputs in Fig. 4 was estimated using the distribution fitting tool of Matlab, and results are shown in Fig. 5. Estimated mean and standard deviation were very close to the values applied, by considering the influence of electrical equipment. According to Figs. 4 and 5, a lower $\sigma$ eliminated the probability of higher instantaneous acceleration by limiting the scattering span.

Using a distribution function with limited scattering span reduces the magnitude of output pulses, while the average (mean) value of pulses remains unaffected.

An analytical survey helps to understand the role of the distribution function in the random vibration test. The navigation equation has the general form of Eq. (6) [4].

$$
\frac{d}{d t} v_{e}=f+g_{l}-\omega_{i e} \times v_{e}
$$

where $v_{e}$ is the vehicle's speed with respect to the earth, $f$ represents the specific acceleration force to which the navigation system is subjected, $g_{l}$ is the local gravity vector and $\omega_{i e}$ is the turn rate of the earth's frame with respect to the inertial frame. This equation may be expressed in inertial, tangent or any other desired frame. Eq. (7) is a discrete form of Eq. (6),
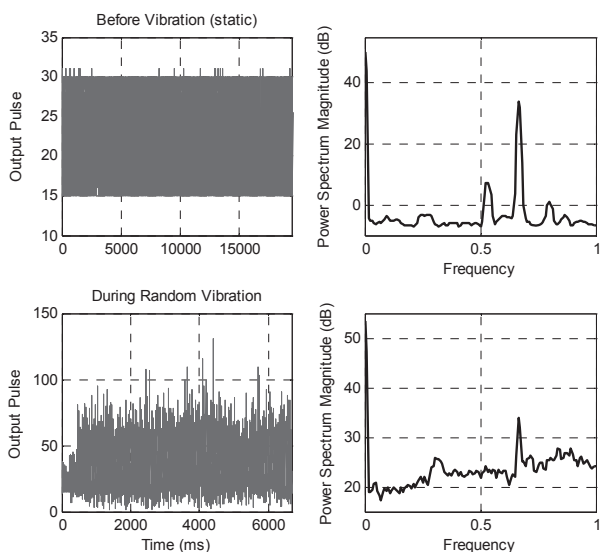

Fig. 3. Effect of random vibration test with profile similar to Fig. 1 on positive channel of gyroscope $X$ 

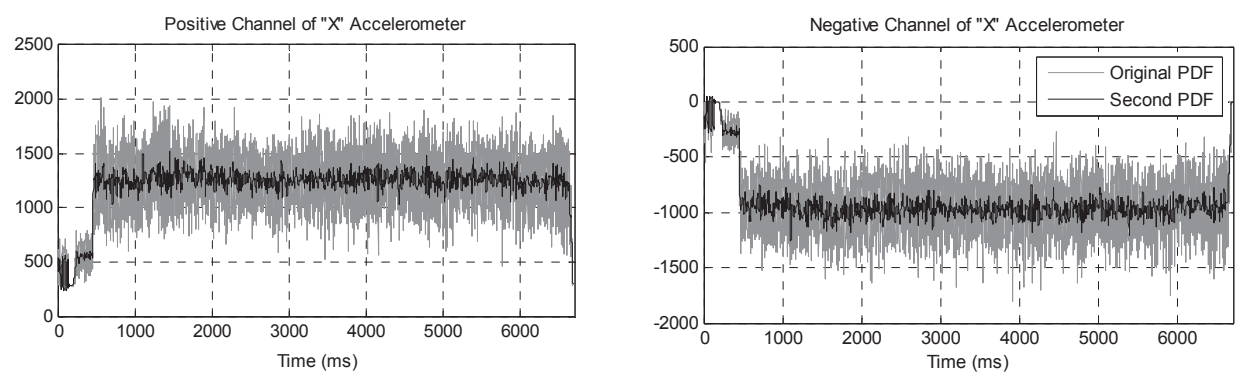

Fig. 4. Effect of random vibration with original and second PDFs in positive channel of accelerometer X

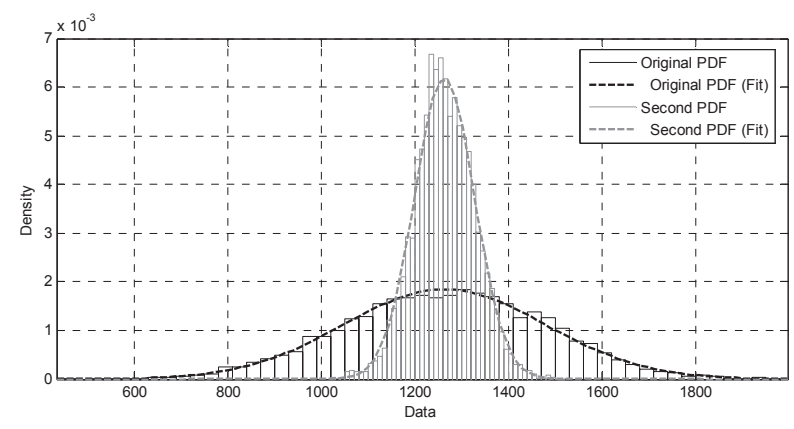

Fig. 5. Estimated PDFs for different output signals of Fig. 4

$$
v_{e_{\text {new }}}=\left(v_{e_{\text {old }}}+\hat{f}_{a}\right)+g_{l}-\omega_{\text {ie }} \times v_{e_{\text {old }}}
$$

where $f_{a}$ is measurement of the sensor in the desired frame. Because measurements provided by the strapdown sensors are in the body frame, pre-multiplication by the proper direction cosine matrix (DCM), is required (Eq. 8).

$$
\hat{f}_{a}=C_{b}^{x} f_{a}
$$

In Eq. (8), $C_{b}^{x}$ is the DCM of the body frame to the desired frame and $f_{a}$ is the compensated output of the sensor, and may be achieved by Eq. (9),

$$
f_{a}=\left(\int_{t_{1}}^{t_{2}} f_{p}\right) \times A+b+K
$$

where $f_{p}$ is the instantaneous output of either accelerometer or gyroscope, and is available only as a summation of pulses in the specific period $\left(t_{2}-t_{1}\right)$. $A$ and $b$ are the scale factor and the bias of the respective sensor. $K$ is a combination of other terms including misalignment errors.
The random vibration directly influences the $f_{p}$, and according to Eqs. (6) to (9), it also affects the navigation results. Changes in the distribution function of the random vibration affects $f_{p}$. However, integration of $f_{p}$ over a specific period of time reduces the effects of distribution function variations. As a result, although the instantaneous output of inertial sensors depends on the PDF of random vibration, the integration decreases the effect of this factor.

\section{Experimental Test}

A random vibration test with the profile shown in Fig. 1 was implemented with original and second PDFs. Data was recorded for about 65 seconds during random vibration (Fig 4). Power spectral density (PSD) describes how the variance of the data is distributed over the frequency components into which the data may be decomposed. PSD estimates for different PDFs in Fig. 4 are illustrated in Fig. 6. This figure

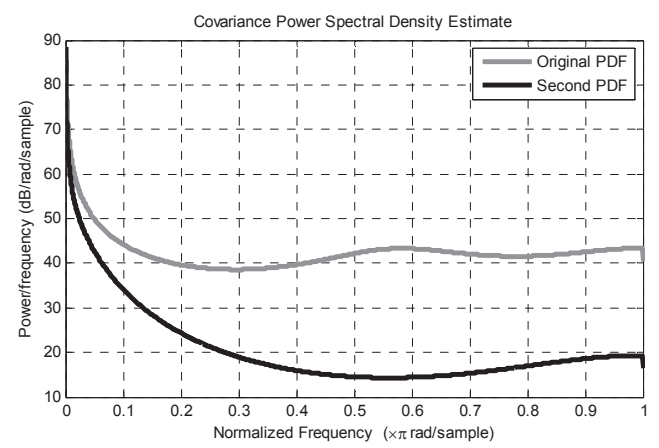

Fig. 6. Covariance power spectral density estimate for signals with original and second distribution function of Fig. 4

Table 1. Numerical comparison between outputs with original and second distribution function

\begin{tabular}{|c|c|c|c|c|}
\hline & Mean & Standard dev. & $\begin{array}{c}\text { RMS Value } \\
\text { Pos. Channel }\end{array}$ & $\begin{array}{c}\text { RMS Value } \\
\text { Neg. Channel }\end{array}$ \\
\hline Original PDF & 1200.786 & 298.367 & 4.372 & 3.376 \\
\hline Second PDF & 1200.802 & 218.763 & 4.313 & 3.306 \\
\hline
\end{tabular}


shows that the vibration PDF influences the power of the signals used in static navigation. A numerical analysis of the signals in Fig. 4 is listed in Table 1. According to Table 1, mean and RMS values are approximately unchanged, while standard deviation decreased significantly.

Using a sliding window with desired length to sum up the signals in Fig. 4, all with different PDFs, reveals that the integration results are very close to each other. It is the same as $\int_{t_{1}}^{t_{2}} f_{p}$ in Eq. (9). The window length is equal to the integration period. On the other hand, variation in RMS value obviously affects $f_{p}$, and subsequently, $v_{e}$.

Figure 7 displays the comprehensive diagram for the testing procedure, as well as the discussed signals. Signals with different PDFs in Fig. 4 will be used for offline static navigation to demonstrate sensitivity to the distribution function. The navigation procedure is identical to real flight navigation.
Strapdown INS was calibrated before the vibration test, using an error model identical to that proposed in [16]. By using calibration coefficients, the compensated outputs of accelerometers $(d v)$ and gyroscopes $(d t)$ will be calculated. After compensation of the earth's angular velocity, dt measures will be used to update the quaternions using fourth-order algorithm as presented in [17]. The $d v$ measures will be used to update the position and velocity of the vehicle in a tangential frame.

The INS experienced 300 seconds of vibration-free condition, and data recorded during this period is used for coarse alignment. Navigation started right at the beginning of vibration and stopped after 75 seconds. Fig. 8 shows position and velocity radial errors. Equality in navigation errors is the result of significant change in vibration PDFs. The INS was examined under different random vibration tests, with different RMS values and equal PDFs. The

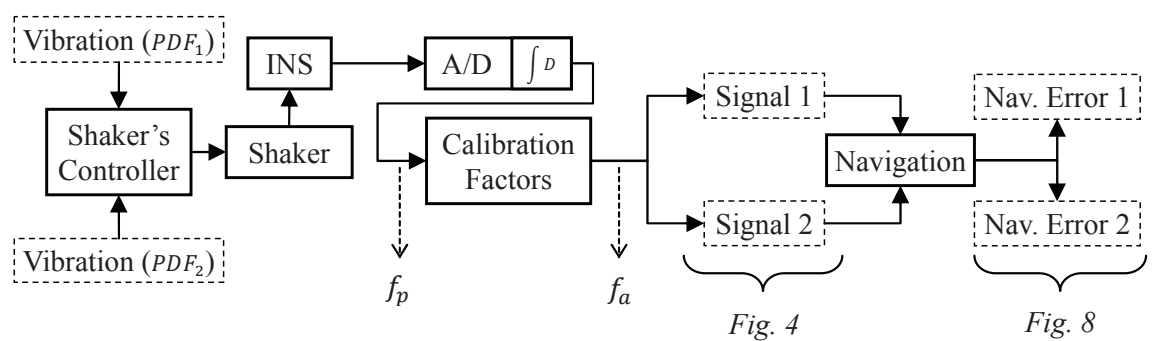

Fig. 7. Diagram of testing procedure
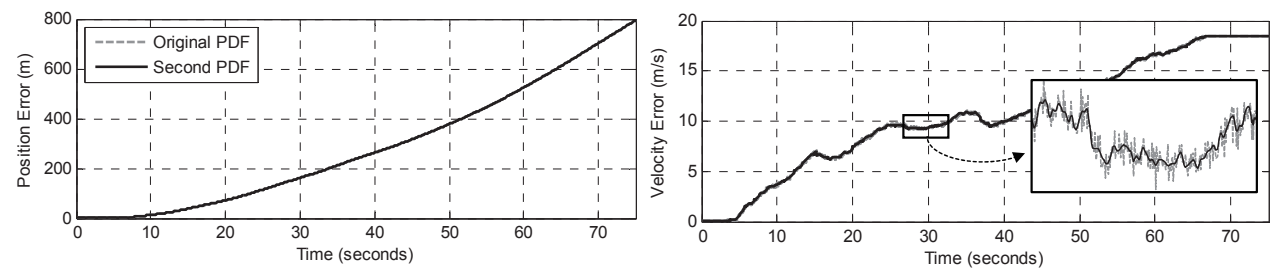

Fig. 8. Comparison between radial error of 75 seconds static navigation, in tests with different PDF
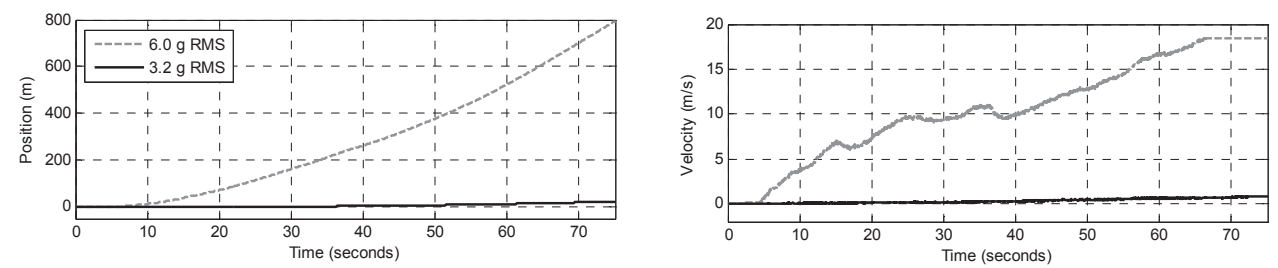

Fig. 9. Comparison between radial error of 75 seconds static navigation, in tests with different RMS

Table 2. Numerical comparison between characteristics of outputs and static navigation with different RMS values

\begin{tabular}{|c|c|c|c|c|c|}
\hline & Mean & Standard dev. & $\begin{array}{c}\text { RMS Value } \\
\text { Pos. Channel }\end{array}$ & $\begin{array}{c}\text { RMS Value } \\
\text { Neg. Channel }\end{array}$ & $\begin{array}{c}\text { Radial Position } \\
\text { Error (meter) }\end{array}$ \\
\hline $6.0 \mathrm{~g}$ RMS & 1200.786 & 298.367 & 4.372 & 3.376 & 793.4 \\
\hline $3.2 \mathrm{~g} \mathrm{RMS}$ & 441.204 & 116.552 & 1.609 & 0.636 & 22.8 \\
\hline
\end{tabular}


numerical comparisons for this new test are summarized in Table 2. The same navigation procedure was applied and the results are illustrated in Fig. 9.

Remarkable improvement in position and velocity error are achieved by decrements in RMS value. Similar tests with different distribution function were performed and the results were similar.

In a random vibration test, the vibration related error was related to only the RMS value, and interestingly not to vibration PDF, despite the fact that it affects the instantaneous outputs.

Previous tests were performed by an INS equipped with three electro-mechanical accelerometers and two dynamically tuned gyroscopes (DTG). Different INS with similar accelerometers and three FOGs were tested using a similar procedure. The test confirmed the previous results. The FOGs contain embedded processors to generate digital values, while former DTGs required external A/D converters. This experiment eliminated the effect of sensor-related behavior and generalized the proposed idea.

\section{Conclusions}

The random vibration test is detailed in relation to INS evaluation. For the first time in a random vibration test of an INS, it has been shown that the magnitude of instantaneous outputs, which is closely related to the distribution function, has a minor effect on navigation error due to the integration of the sensor's output. On the other hand, the RMS value of the random vibration test determines the major errors in position and velocity estimations. These claims were confirmed using analytical methods and experimental tests. Therefore, the most effective method to reduce vibration related error in strapdown inertial navigation is one that reduces RMS value sensed by inertial sensors. This could be applied in the form of a well-engineered damper with either mechanical or electrical implementation.

\section{References}

[1] K. R. Curey, M. E. Ash, L. O. Thielman, and C. H. Baker, Proposed IEEE Inertial Systems Terminology Standards and Other Inertial Sensor Standards, 2004.

[2] C. Zaiss. IMU Design for High Vibration Environments with Special Consideration for Vibration Rectification, MSc. Thesis, Department of Mechanical and Manufacturing
Engineering, Calgary, Alberta, 2012.

[3] A. Bose, S. Puri, and P. Banerjee, Modern Inertial Sensors and Systems. Prentice-Hall Publications, New Delhi, India, 2008.

[4] D. H. Titterton, J. L. Weston, Strapdown Inertial Navigation Technology, 2nd Edition, Peter Peregrinus Ltd, Chapter 7, 2005.

[5] D. A. Lorenzini, Testing of Precision Inertial Gyroscopes, Advisory Group for Aerospace Research and Development, 1973.

[6] D. S. Steinberg, Vibration Analysis for Electronic Equipment, Third Edition, Wiley Inter-science Publication, Chapter 14, 2000.

[7] P. Ripka, and A. Tipek, Modern Sensors Handbook. Wiley-ISTE Pub., Great Britain, Chapter 5, 2007.

[8] D. E. Newland, An introduction to random vibrations and spectral analysis, 2nd edition, Longman, London, 1984.

[9] J. V. Baren, What is Random Vibration Testing? , Sound and Vibration magazine, Instrument Reference Issue, February 2012.

[10] IEEE Recommended Practice for Inertial Sensor Test Equipment, Instrumentation, Data Acquisition, and Analysis, IEEE std-1554-2005, 2012.

[11] K. Y. Chang, Deep Space 1 Spacecraft Vibration Qualification Testing. Institute of Environmental Sciences and Technology, 46th Annual Technical Meeting, Providence, 2000.

[12] X. Hao, M. Li, and H. Jia, Random Vibration Analysis on the Support of Strapdown Inertial Navigation System, Int. Conference on Electrical and Control Engineering, 2011, pp 4025-4028.

[13] C. Shen, X. Chen, J. Yu, and J. Wu, Random drift modeling and compensation for fiber optic gyroscope under vibration, IEEE Instrumentation and Measurement Technology Conference, 2011.

[14] F. Sun, Q. Sun, L. Wu, and W. Sun, Error compensation for FOG SINS under vibration based on Elman Neural Network, International Conference on Mechatronics and Automation, 2009, pp 302-307

[15] L. Gang, Y. Jie, W. Lixin, G. Jiang, and W. Xiaomei, Detecting and Extracting Vibration Disturb in IMU Testing in Field. 2nd Int. Conference on Computer Engineering and Technology, 2010, pp 516-518.

[16] A. Abdoli, and S. H. Taghavi, Calibration of Strapdown Inertial Measurement Units by Linear Neural Networks, the 13th Iranian Aerospace Society Conference, Tehran, 2014.

[17] J. A. Farrell, Aided Navigation; GPS with High Rate Sensors, McGraw-Hill, 2008, Page 508-509. 